\title{
Estoque de Serapilheira e Atividade Microbiana em Solo sob Sistemas Agroflorestais*
}

\author{
Miguel Seabra Correa da Silva ${ }^{1}$, Eliane Maria Ribeiro da Silva ${ }^{2}$, \\ Marcos Gervasio Pereira ${ }^{3}$, Cristiane Figueira da Silva ${ }^{3}$ \\ ${ }^{1}$ Secretaria de Pesca e Agricultura de Paraty, Paraty/RJ, Brasil \\ ${ }^{2}$ Embrapa Agrobiologia, Laboratório de Micorrizas, Seropédica/RJ, Brasil \\ ${ }^{3}$ Departamento de Solos, Universidade Federal Rural do Rio de Janeiro, Seropédica/RJ, Brasil \\ *Parte da Dissertação apresentada pelo primeiro autor ao Curso de Pós-graduação em Agronomia - \\ Ciência do Solo (CPGA-CS) da Universidade Federal Rural do Rio de Janeiro (UFRRJ).
}

\begin{abstract}
RESUMO
Este estudo objetivou avaliar o efeito de dois tipos de manejo agroflorestal (SAFs 1 e 2) sobre os estoques de serapilheira, as características químicas do solo e a atividade e a biomassa microbiana do solo (BMS), comparando-os a uma área com agricultura anual (AgAn) e uma mata secundária no município de Paraty-RJ. O manejo de poda nos SAFs promoveu aumento nos teores e/ou nos estoques de nitrogênio, fósforo, potássio e magnésio da serapilheira, levando à condição de semelhança aos estoques da mata e de superioridade em relação aos estoques da AgAn. No verão, o carbono da BMS e a respiração basal foram mais elevados na área de mata, enquanto que, em ambas as estações, inverno e verão, não houve diferença entre os SAFs e a área de AgAn. O SAF-2 apresentou elevado quociente microbiano no inverno, demonstrando maior sensibilidade dos microrganismos às mudanças de cobertura vegetal do que aos atributos químicos do solo.
\end{abstract}

Palavras-chave: agroecologia, indicadores microbiológicos, respiração do solo.

\section{Litter Stock and Soil Microbial Activity in Agroforestry Systems}

\begin{abstract}
This study aimed to evaluate the effect of two agroforestry management systems (AFS 1 and 2) on the stocks of litter, soil chemical properties, soil activity, and soil microbial biomass (SMB), comparing them to an annual agriculture area (AnAg) and a secondary forest area in the municipality of Paraty, State of Rio de Janeiro. The pruning management of the AFSs has promoted increased levels and/or stocks of nitrogen, phosphorus, potassium and magnesium in the litter, taking the systems to the condition of similarity when compared to the secondary forest and superiority in relation to the stock observed in the AnAg area. The carbon content from the SMB and the soil basal respiration did not vary between the AFS and the AnAg area throughout the year. In contrast, the forest area presented the highest values of these variables combined with the total organic carbon content in the summer. AFS-2 showed high microbial quotient in the winter, presenting higher sensitivity of microorganisms to changes in vegetation cover than in soil chemical properties.
\end{abstract}

Keywords: agroecology, microbial indicators, soil respiration. 


\section{INTRODUÇÃO}

Os sistemas agroflorestais (SAFs) são uma combinação integrada de árvores, arbustos, cultivos agrícolas e animais na mesma área, de maneira simultânea ou sequencial, que buscam a otimização da agregação de valores socioeconômico-culturais e ambientais, visto que os mesmos apresentam potencial para constituírem uma modalidade sustentável de uso e manejo dos recursos naturais (Assis-Júnior et al., 2003). Estes sistemas são difundidos como uma prática na qual o consórcio de culturas agrícolas com espécies arbóreas resulta na melhoria das condições físicas e químicas dos solos, em razão da diminuição da erosão e do aumento no aporte de matéria orgânica no solo (Reinert, 1998; Mendonça et al., 2001). A utilização desta prática é indicada para a recuperação de áreas degradadas e para a preservação da área de entorno de reservas ambientais. Estes sistemas não restauram por completo a estrutura e a biodiversidade de comunidades florestais, mas podem auxiliar a recuperação das funções essenciais para a sustentabilidade do agroecossistema, como a ciclagem de nutrientes, além de gerar uma renda ou produção de subsistência ao agricultor rural (MacDicken \& Vergara, 1990).

Além da recuperação de funções ecológicas que garantem a sustentabilidade do agroecossistema, as agroflorestas apresentam grande potencial para o sequestro de carbono atmosférico em tecido vegetal ou matéria orgânica no solo (Albrecht \& Kandji, 2003).

A respiração do solo representa a soma total da atividade metabólica do solo e os processos biológicos responsáveis por essa atividade são a respiração microbiana, a respiração da fauna e a respiração das raízes (Lundegardh, 1927). Da totalidade de carbono fixado pelas plantas (cerca de 60 a $61 \mathrm{PgC}$ são retirados da atmosfera anualmente), $60 \mathrm{PgC}$ retornam para a atmosfera por meio dos tecidos vivos e da decomposição da matéria orgânica na superfície do solo (Schlesinger, 1997), sendo a emissão de $\mathrm{CO}_{2}$ do solo a principal via de retorno de carbono para a atmosfera. Desta forma, as modificações na magnitude desta emissão, provocadas pelo uso da terra, podem acarretar grandes alterações nas concentrações de $\mathrm{CO}_{2}$ atmosférico (Schlesinger \& Andrews, 2000).

A contribuição das raízes para a respiração total do solo varia de $10 \%$ a $90 \%$, dependendo da estação do ano e da vegetação; estudos de longa duração indicam as contribuições médias de $45 \%$ e $60 \%$, respectivamente, em áreas florestadas e abertas (Hanson et al., 2000). Contudo, os microrganismos do solo, nas suas atividades metabólicas de ciclagem de nutrientes e de energia, são os principais responsáveis pela respiração total do solo. Por isso, a determinação da biomassa microbiana do solo (BMS) é importante e consiste numa estimativa da parte viva da matéria orgânica menor que $5.000 \mu \mathrm{m}^{3}$, composta por bactérias, actinobactérias, fungos, algas e toda a comunidade microbiana (Jenkinson \& Ladd, 1981). A estimativa da BMS foi sugerida como um parâmetro sensível às mudanças iniciais no processo de transformação de matéria orgânica do solo, sendo um indicador útil para avaliar alterações em ecossistema com interferência antrópica (Jenkinson \& Rainer, 1977; Powlson et al., 1987).

Anderson \& Domsch (1985) propuseram a razão entre a respiração basal do solo e o carbono da BMS (quociente metabólico microbiano $=q \mathrm{CO}_{2}$ ) como um indicador das mudanças da atividade microbiana após distúrbios. Este índice indica se a biomassa microbiana está sendo eficiente (menores valores de $q \mathrm{CO}_{2}$ ) na utilização dos recursos, incorporando carbono em sua biomassa, ou se o carbono está sendo perdido rapidamente na forma de $\mathrm{CO}_{2}$ (maiores valores de $q \mathrm{CO}_{2}$ ). Ou seja, indica a eficiência de uso do substrato pela microbiota do solo (Silva et al., 2007).

O município de Paraty-RJ tem população de 29.544 habitantes, dos quais $52 \%$ estão localizados em estabelecimentos rurais menores que 50 hectares (Prefeitura Municipal de Paraty, 2000), conferindo à agricultura do município um caráter familiar. A prática agrícola predominante é o sistema tradicional caiçara, na qual a área florestada a ser cultivada é derrubada, queimada e culturas anuais são introduzidas. O município apresenta cinco unidades de conservação da Mata Atlântica e a maioria das propriedades rurais se localiza dentro ou na área de entorno destas unidades, tornando o desenvolvimento agrícola na região uma 
ameaça à sua conservação. Por tudo isso, o caráter conservacionista dos sistemas agroflorestais torna essa prática indicada para a situação de Paraty.

O presente estudo teve como objetivo avaliar o reflexo do manejo agroflorestal de dois diferentes SAFs sobre os atributos químicos e biológicos do solo, e da serapilheira, comparando com áreas de agricultura anual (monocultivo de mandioca) e um fragmento de mata secundária natural no município de Paraty-RJ.

\section{MATERIAL E MÉTODOS}

O estudo foi realizado na comunidade quilombola do Campinho da Independência, localizada no distrito de Paraty-Mirim, município de Paraty-RJ, nas coordenadas geográficas $44^{\circ} 42^{\prime} \mathrm{W}$ e $23^{\circ} 17^{\prime} \mathrm{S}$, e altitude de $60 \mathrm{~m}$. A comunidade está localizada na parte central da área de proteção ambiental (APA) do Cairuçu e às margens do Rio Paraty-Mirim e da BR 101. As vegetações nativas remanescentes são florestas de encosta do tipo ombrófila densa submontana (Brasil, 1983).

O estudo foi desenvolvido numa área onde ocorrem as seguintes coberturas vegetais: dois diferentes sistemas agroflorestais (SAFs 1 e 2, com área de $400 \mathrm{~m}^{2}$ cada), uma área de floresta secundária e uma área com agricultura anual (monocultura de mandioca, com área de $600 \mathrm{~m}^{2}$ ). Os SAFs foram implantados em uma propriedade rural em fevereiro de 2003, tendo como cultura de principal interesse econômico o palmito de pupunheira (Bactris gasipaes Kunth.). Ambos os SAFs apresentam espécies frutíferas de ciclo curto, como a banana variedade ouro (Musa sp.) e o mamão papaya (Carica papaya L.); espécies alimentares de subsistência, como o feijão-preto (Phaseolus vulgaris L.), o milho (Zea mays L.) e a mandioca (Manihot esculenta L.), e espécies de adubação verde, como o feijão-de-porco (Canavalia ensiformis (L.) DC.), o feijão-guandu (Cajanus cajans (L.) Millsp.) e a crotalária (Crotalaria juncea L.).

As unidades experimentais SAF-1 e SAF-2 se diferenciam na composição de espécies frutíferas de ciclo longo, sendo o abiu (Pouteria caimito Radlk.) e a jaca (Artocarpus heterophyllus Lam.) no SAF-1, e a graviola (Anona muricata L.) e a carambola (Averrhoa carambola L.) no SAF-2. Os SAFs também se diferem na composição de espécies renovadoras de fertilidade. No SAF-1, estão presentes o ingá (Inga $s p$ ) e a embira-de-sapo (Lonchocarpus guilleminianus (Tul.) Malme), e no SAF-2 estão presentes o urucum (Bixa orelana L.) e o pau-viola (Cytharexyllum myrianthum Cham.). As composições de espécies clímax e de espécies de uso potencial para sementes, óleos e madeira de lei também diferem nos dois SAFs, sendo que no SAF-1 estão presentes as leguminosas guapuruvu (Schizolobium parahyba (Vell.) Blake), araribá (Centrolobium tomentosum Guill. ex Benth.), jatobá (Hymenaea courbaril L.) e copaíba (Copaifera langsdorffii Desf.), e no SAF-2 estão presentes as não leguminosas cedro (Cedrela fissilis Vell.), canela (Nectrandra lanceolata Nees et Mart. ex Ness.), jequitibá (Cariniana legalis (Mart.) Kuntze) e a bicuíba (Virola bicuhyba (Schott) Warb.).

A avaliação do incremento de matéria orgânica e nutrientes proporcionado pelo manejo agroflorestal foi realizada a partir de análises quantitativas e de composição química das camadas de serapilheira, sendo que os sistemas agroflorestais foram avaliados antes e depois do manejo da poda das espécies utilizadas como adubação verde e renovadoras de fertilidade. A quantidade de serapilheira acumulada sobre a superfície do solo foi estimada sobre um gabarito de $0,5 \times 0,5 \mathrm{~m}$, sendo realizadas cinco amostragens em cada tratamento. As amostras de serapilheira seca foram pesadas para determinação da biomassa e, em seguida, o material foi triturado para a análise dos teores de nutrientes, segundo Embrapa (1997). O delineamaneto experimental foi o inteiramente casualizado.

Para a avaliação das características químicas e biológicas do solo, foram coletadas cinco amostras compostas, formadas a partir de dez amostras simples, na camada de $0-10 \mathrm{~cm}$. Após a coleta, o solo foi seco ao ar, peneirado e analisado quanto aos teores de matéria orgânica, carbono orgânico total (COT), nitrogênio total $(\mathrm{NT})$, alumínio $\left(\mathrm{Al}^{+3}\right)$, cálcio $\left(\mathrm{Ca}^{+2}\right)$, magnésio $\left(\mathrm{Mg}^{+2}\right)$, fósforo $(\mathrm{P})$, potássio $\left(\mathrm{K}^{+2}\right)$ e pH em água (Embrapa, 1997). A caracterização microbiológica foi realizada a partir das seguintes variáveis: carbono da biomassa microbiana (C-BMS) avaliado pelo método de fumigação-extração de Vance et al. (1987) e Bartlett \& Ross (1988); respiração basal do solo (RBS), de acordo com (Jenkinson \& 
Powlson, 1976); o quociente metabólico $\left(q \mathrm{CO}_{2}\right)$ foi obtido pela razão entre a RBS por unidade de C-BM (Anderson e Domsch, 1985, 1990) e o quociente microbiano ( $q \mathrm{MIC})$, pela razão entre o C-BMS e o COT do solo.

A densidade de raízes foi estimada por meio do peneiramento e da catação manual de amostras de solo da camada de $0-10 \mathrm{~cm}$. As raízes foram classificadas em raízes finas (menores que $1 \mathrm{~mm}$ ) e raízes grossas (maiores que $1 \mathrm{~mm}$ ). Após a separação, as raízes foram lavadas em água corrente e secas em estufa de $60^{\circ} \mathrm{C}$ por 48 horas até peso constante para a determinação da massa seca.

Para a determinação da contribuição relativa de raízes e da serapilheira para a respiração total do solo, medições de emissão de $\mathrm{CO}_{2}$ foram realizadas em campo pelo método da cromatografia gasosa. Em cada área, cinco câmaras circulares de $30 \mathrm{~cm}$ de diâmetro foram sobrepostas ao solo e amostras de gases do interior das câmaras foram retiradas com seringas em intervalos de um minuto. As taxas de emissão foram estimadas para cada câmara. Sob cada câmara, foram quantificadas a serapilheira e as raízes na profundidade de $0-10 \mathrm{~cm}$ para correlacionar com a emissão total de $\mathrm{CO}_{2}$, de acordo com técnica de regressão proposta por Kucera \& Kirkham (1971).

Os sistemas agroflorestais em estudo foram implantados como unidades demonstrativas no Quilombo do Campinho e em outras comunidades em Paraty, e por isso não apresentam delineamento experimental com blocos de repetições distribuídas ao acaso. Desta forma, uma análise de variância se torna inadequada e se faz necessária a comparação de médias duas a duas pelo teste $t$ de Bonferroni (Bailey, 1977). As análises univariadas foram feitas com o auxílio do programa Sisvar (Ferreira, 2003).

\section{RESULTADOS E DISCUSSÃO}

\subsection{Deposição de serapilheira durante o manejo agroflorestal}

Os sistemas agroflorestais (SAFs) foram avaliados antes e depois do manejo para a poda das leguminosas destinadas à adubação verde e das espécies renovadoras de fertilidade. Antes da poda, a área de mata secundária apresentou o maior estoque de serapilheira entre as áreas (Tabela 1), diferindo significativamente da quantidade detectada na agricultura anual (AgAn) e do SAF-2, tendo apresentado os maiores valores. Após a poda, o SAF2 apresentou um incremento (190\%) de matéria orgânica (MO) na serapilheira, sendo este valor estatisticamente significativo. Na ocasião, os estoques de serapilheira dos SAFs 1 e 2 foram estatisticamente iguais aos da área de mata.

Os teores de nutrientes adicionados via serapilheira são apresentados na Tabela 1. Em relação aos teores de $\mathrm{N}$, a serapilheira da área de mata apresentou uma média estatisticamente maior em comparação à área do SAF-1 antes da poda e também em relação à área de AgAn. Após a poda, o teor médio de $\mathrm{N}$ na área do SAF-2 se elevou significativamente, se igualando ao da área de mata

Tabela 1. Teores e estoques de nutrientes $\left(\mathrm{g} \mathrm{kg}^{-1} \mathrm{e} \mathrm{g} \mathrm{m}^{-2}\right)$ na serapilheira e estoque de serapilheira sobre os solos de sistemas agroflorestais (SAF), mata secundária e agricultura anual (AgAn).

Table 1. Tenors and stocks of nutrients $\left(\mathrm{g} \mathrm{kg}^{-1}\right.$ and $\mathrm{g} \mathrm{m}^{-2}$ ) and stock of litter on the soil of agroforestry (AFS), secondary forest and annual agricultural (AnAg).

\begin{tabular}{|c|c|c|c|c|c|c|c|c|c|c|c|}
\hline \multirow{2}{*}{ Áreas } & $\mathbf{N}$ & $\mathbf{P}$ & $\mathbf{K}$ & $\mathrm{Ca}$ & $\mathrm{Mg}$ & Serapilheira & $\mathbf{N}$ & $\mathbf{P}$ & $\mathbf{K}$ & $\mathrm{Ca}$ & $\mathrm{Mg}$ \\
\hline & \multicolumn{5}{|c|}{$\mathrm{g} \mathrm{kg}^{-1}$} & \multicolumn{6}{|c|}{$\mathrm{g} \mathrm{m}^{-2}$} \\
\hline \multicolumn{12}{|c|}{ Antes do manejo } \\
\hline SAF-1 & $13,30^{c}$ & $0,98^{\mathrm{b}}$ & $0,19^{c}$ & $8,36^{\mathrm{a}}$ & $1,81^{\mathrm{ab}}$ & $442,40^{\mathrm{bc}}$ & $5,61^{\mathrm{c}}$ & $0,43^{b}$ & $0,08^{\mathrm{b}}$ & $3,69^{\mathrm{ab}}$ & $0,80^{\mathrm{b}}$ \\
\hline SAF-2 & $15,70^{\mathrm{bc}}$ & $0,86^{\mathrm{b}}$ & $0,20^{c}$ & $10,47^{\mathrm{a}}$ & $1,91^{\mathrm{ab}}$ & $290,16^{c}$ & $4,39^{c}$ & $0,25^{\mathrm{b}}$ & $0,06^{\mathrm{b}}$ & $3,04^{\mathrm{ab}}$ & $0,55^{\mathrm{b}}$ \\
\hline AgAn & $15,10^{c}$ & $0,31^{\mathrm{b}}$ & $0,02^{\mathrm{c}}$ & $5,07^{\mathrm{a}}$ & $0,93^{\mathrm{b}}$ & $193,84^{c}$ & $2,83^{\mathrm{c}}$ & $0,06^{\mathrm{b}}$ & $0,004^{\mathrm{b}}$ & $0,98^{\mathrm{b}}$ & $0,18^{\mathrm{b}}$ \\
\hline Mata & $22,90^{\mathrm{ab}}$ & $0,48^{\mathrm{b}}$ & $0,06^{c}$ & $11,33^{\mathrm{a}}$ & $1,64^{\mathrm{ab}}$ & $689,84^{\mathrm{ab}}$ & $15,74^{\mathrm{ab}}$ & $0,33^{\mathrm{b}}$ & $0,04^{\mathrm{b}}$ & $7,81^{\mathrm{a}}$ & $1,13^{\mathrm{ab}}$ \\
\hline \multicolumn{12}{|c|}{ Após o manejo } \\
\hline SAF-1 & $19,10^{\mathrm{abc}}$ & $1,91^{\mathrm{a}}$ & $6,10^{\mathrm{a}}$ & $8,83^{\mathrm{a}}$ & $2,65^{\mathrm{a}}$ & $739,60^{\mathrm{ab}}$ & $13,05^{\mathrm{b}}$ & $1,42^{\mathrm{a}}$ & $4,51^{\mathrm{a}}$ & $6,53^{\mathrm{ab}}$ & $1,96^{\mathrm{a}}$ \\
\hline SAF-2 & $23,50^{\mathrm{a}}$ & $1,71^{\mathrm{a}}$ & $2,95^{\mathrm{b}}$ & $8,03^{a}$ & $2,31^{\mathrm{ab}}$ & $842,88^{a}$ & $19,58^{\mathrm{a}}$ & $1,44^{\mathrm{a}}$ & $2,49^{a}$ & $6,77^{\mathrm{ab}}$ & $1,95^{\mathrm{a}}$ \\
\hline
\end{tabular}

Letras iguais na coluna indicam que não há diferença entre as médias pelo teste $t$ de Bonferroni. 
e sendo superior à área de AgAn, enquanto para a área de SAF-1, não foram verificadas diferenças significativas. A comparação das médias de estoque de $\mathrm{N}$ na serapilheira mostrou que a área de mata possui uma quantidade significativamente superior às outras áreas, antes do manejo. Com a poda, o estoque de $\mathrm{N}$ na serapilheira dos SAFs se igualou estatisticamente à mata, sendo que na área de SAF-2 o estoque foi superior ao da área de SAF-1.

Os teores e estoques de Pede $\mathrm{K}^{+}$foram semelhantes em todas as áreas antes da poda. Com a poda, tanto os teores quanto os estoques destes elementos nas áreas de SAFs 1 e 2 se elevaram significativamente, e formaram um grupo estatisticamente maior do que as outras áreas. Foi verificado que os teores de $\mathrm{K}^{+}$na área de SAF-1 foram superiores àqueles encontrados na área de SAF-2 depois do manejo (Tabela 1).

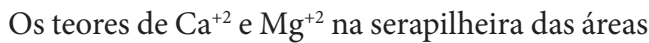
de estudo não apresentaram diferença estatística, antes da poda. No entanto, com a deposição proporcionada pela poda, os teores de $\mathrm{Mg}^{+2}$ se elevaram na serapilheira das áreas de SAFs, sendo o teor na área do SAF-1 superior ao da área de AgAn. Os estoques de $\mathrm{Mg}^{+2}$ na serapilheira também não variaram entre as áreas antes do manejo. Por outro lado, a poda promoveu nas áreas dos SAFs 1 e 2 um incremento da ordem de $145 \%$ e $254 \%$ no estoque deste elemento, respectivamente, em relação à situação dos SAF's antes do manejo e, de $983 \%$ em relação à área de AgAn, se igualando estatisticamente à área de mata (Tabela 1).

\subsection{Carbono orgânico total e macronutrientes do solo}

As análises das características químicas dos solos mostraram que, no inverno, as áreas de mata e o SAF-1 apresentaram maiores teores de carbono orgânico total (COT) e nitrogênio total (NT), em comparação à área de SAF-2 (Tabela 2). Quando se compararam os teores de COT e NT no solo, entre as áreas, no verão, observou-se que a mata apresentou os maiores valores em relação às demais áreas. Quanto à sazonalidade, verificou-se um aumento significativo nos teores de COT nas áreas de SAF-2, AgAn e de mata, do inverno para o verão. Este padrão também foi observado nas áreas de AgAn e de mata, para o NT, o que pode ser decorrente da maior decomposição do material aportado ao solo, tendo em vista as maiores precipitações pluviométricas que ocorreram no verão, as quais favorecem a atividade dos organismos do solo.

Em relação ao $\mathrm{pH}$, as áreas de SAFs 1 e 2 apresentaram solos menos ácidos em comparação à área de mata, em ambas as estações (Tabela 2). Verificou-se redução nos valores de $\mathrm{pH}$, do inverno para o verão, nas áreas de SAF-1, AgAn e mata. Em estudos em áreas sob uso agroflorestal e em florestas remanescentes adjacentes no norte de Rondônia, Menezes et al. (2008) observaram que os valores de $\mathrm{pH}$ e os teores de $\mathrm{Ca}^{2+}$ e $\mathrm{Mg}^{2+}$ foram mais altos nos plantios agroflorestais quando comparados aos solos das florestas remanescentes. Neste estudo, os teores

Tabela 2. Caracterização química da camada $0-10 \mathrm{~cm}$ do solo de dois sistemas agroflorestais (SAFs 1 e 2), mata secundária e agricultura anual (AgAn), em duas estações (inverno-I; verão-V).

Table 2. Chemical characterization of 0-10 layer of two agroforestry systems (AFS 1 e 2), secondary forest e annual agriculture (AnAg), in two seasons (winter-W; summer-S). S = season; AgAn = annual agriculture.

\begin{tabular}{|c|c|c|c|c|c|c|c|c|c|}
\hline \multirow{2}{*}{ Áreas } & $\mathbf{F}$ & $\mathrm{COT}$ & $\mathbf{N}$ & \multirow{2}{*}{$\mathbf{p H}$} & Al & $\mathrm{Ca}$ & $\mathrm{Mg}$ & $\mathbf{P}$ & $\mathrm{K}$ \\
\hline & $\mathbf{E}$ & \multicolumn{2}{|c|}{$\mathrm{g} \mathrm{kg}^{-1}$} & & \multicolumn{3}{|c|}{$\mathrm{cmol}_{\mathrm{c}} \mathrm{dm}^{-3}$} & \multicolumn{2}{|c|}{$\mathrm{mg} \mathrm{dm}^{-3}$} \\
\hline \multirow[t]{2}{*}{ SAF 1} & I & $26,5^{\mathrm{aA}}$ & $3,3^{\mathrm{abA}}$ & $5,6^{\mathrm{aA}}$ & $0,07^{\mathrm{bB}}$ & $3,7^{\mathrm{aA}}$ & $1,53^{\mathrm{aA}}$ & $11,0^{\mathrm{aA}}$ & $267^{\mathrm{aA}}$ \\
\hline & V & $26,9^{\mathrm{bA}}$ & $3,3^{\mathrm{bcA}}$ & $5,0^{\mathrm{aB}}$ & $0,48^{\mathrm{aA}}$ & $2,7^{\mathrm{abA}}$ & $1,04^{\mathrm{bA}}$ & $7,0^{\mathrm{bB}}$ & $51^{\mathrm{abB}}$ \\
\hline \multirow[t]{2}{*}{ SAF 2} & I & $20,5^{\mathrm{bB}}$ & $2,4^{\mathrm{Ba}}$ & $5,4^{\mathrm{aA}}$ & $0,33^{\mathrm{bA}}$ & $1,8^{\mathrm{aA}}$ & $1,3^{\mathrm{aA}}$ & $6,3^{\mathrm{aA}}$ & $73^{\mathrm{bA}}$ \\
\hline & V & $24,1^{\mathrm{bA}}$ & $2,7^{\mathrm{cA}}$ & $5,1^{\text {aA }}$ & $0,44^{\mathrm{aA}}$ & $2,24^{\mathrm{abA}}$ & $1,14^{\mathrm{bA}}$ & $7,0^{\mathrm{bA}}$ & $42,6^{\mathrm{bB}}$ \\
\hline \multirow[t]{2}{*}{ AgAn } & I & $25,5^{\mathrm{abB}}$ & $3,2^{\mathrm{abB}}$ & $5,2^{\mathrm{abA}}$ & $1,03^{\mathrm{aA}}$ & $1,7^{\mathrm{aA}}$ & $1,43^{\mathrm{aA}}$ & $7,6^{\mathrm{aA}}$ & $88^{\mathrm{bA}}$ \\
\hline & V & $30,6^{\mathrm{bA}}$ & $3,7^{\mathrm{bA}}$ & $4,8^{\mathrm{aB}}$ & $0,96^{\mathrm{aA}}$ & $1,76^{\mathrm{bA}}$ & $1,04^{\mathrm{bA}}$ & $11,0^{\mathrm{abA}}$ & $151,8^{\mathrm{aA}}$ \\
\hline \multirow[t]{2}{*}{ Mata } & I & $29,1^{\mathrm{aB}}$ & $3,8^{\mathrm{aB}}$ & $4,9^{\mathrm{bA}}$ & $1,6^{\mathrm{aA}}$ & $1,7^{\mathrm{aA}}$ & $1,17^{\mathrm{aA}}$ & $8,3^{\mathrm{aB}}$ & $110^{\mathrm{bA}}$ \\
\hline & V & $44,0^{\mathrm{aA}}$ & $5,9^{\mathrm{aA}}$ & $4,5^{\mathrm{bB}}$ & $1,02^{\mathrm{aA}}$ & $3,24^{\mathrm{aA}}$ & $1,72^{\mathrm{aA}}$ & $15,0^{\mathrm{aA}}$ & $91,4^{\mathrm{abA}}$ \\
\hline
\end{tabular}

$\mathrm{E}=$ estação; AgAn = agricultura anual; COT = Carbono Orgânico Total. Letras minúsculas iguais numa mesma coluna representam igualdade estatística entre os tratamentos na mesma estação, enquanto letras maiúsculas iguais numa mesma coluna representam igualdade estatística entre as estações no mesmo tratamento segundo o teste t com correção de Bonferroni ( $\mathrm{p}<0,05)$. 
de $\mathrm{Ca}^{+2}, \mathrm{Mg}^{+2}$ e $\mathrm{P}$ não apresentaram diferenças entre as áreas dos SAFs 1 e 2. No entanto, no inverno, estes sistemas apresentaram os teores de $\mathrm{Mg}^{+2}$ e $\mathrm{P}$ inferiores aos observados na área de mata. Os teores de $\mathrm{Ca}^{+2}$ e $\mathrm{Mg}^{+2}$ não apresentaram incremento significativo do inverno para o verão, sendo que a área sob mata apresentou teores de $\mathrm{Ca}^{+2}$ superiores à área de AgAn e teores de $\mathrm{Mg}^{+2}$ superiores às demais áreas, no verão.

Os teores de $\mathrm{K}^{+}$nas áreas dos SAFs 1 e 2 diminuíram do inverno para o verão e em ambas as épocas, sendo similares à mata, à exceção da área do SAF-1 no inverno, que foi estatisticamente superior. Em seus estudos em áreas de fragmentos florestais com diferentes estádios de recuperação, Menezes et al. (2008) não verificaram diferenças significativas para os conteúdos de $\mathrm{P}$ e $\mathrm{K}^{+}$entre sistema agroflorestal e florestas remanescentes adjacentes, indicando que os sistemas agroflorestais preservaram algumas propriedades químicas do solo com valores similares ao da área de floresta natural durante o período considerado.

Os teores de $\mathrm{Al}^{+3}$ encontrados nas áreas de SAFs 1 e 2 foram inferiores aos observados nas áreas de AgAn e mata, tendo esta última apresentado um teor considerado tóxico. As concentrações de $\mathrm{Al}^{+3}$ se elevaram do inverno para o verão apenas na área de SAF-1, não havendo diferença nos teores deste elemento entre as diferentes áreas, no verão.
Avaliando a fertilidade do solo em SAFs, com diferentes arranjos e mata primária, Cidin et al. (2009) observaram que os teores de $\mathrm{P}^{+} \mathrm{K}^{+} \mathrm{Al}^{+3}$ não variaram significativamente entre estas áreas.

\subsection{Atividade e carbono da biomassa microbiana}

Na Tabela 3, são apresentados o carbono da biomassa microbiana (C-BMS), a respiração basal do solo (RBS) e os quocientes microbiano ( $q \mathrm{MIC}$ ) e metabólico $\left(q \mathrm{CO}_{2}\right)$ do solo das áreas em estudo. Verifica-se que, no inverno, as concentrações C-BMS e RBS foram semelhantes em todas as áreas. No verão, ambas as variáveis apresentaram valores mais elevados na área de mata em relação às demais, sendo que apenas a RBS, na mata, variou entre as estações, apresentando maiores valores no verão. Para os SAFs, verificaram-se teores de C-BMS e RBS intermediários entre os da área de mata e da área de AgAn, diferentemente do que se encontrou para a fertilidade do solo, mostrando que estes indicadores são mais responsivos a modificações na estrutura da vegetação. Avaliando a atividade microbiana do solo em diferentes ecossistemas, Assis-Júnior et al. (2003) também observaram, em áreas de SAFs, taxas de RBS intermediárias entre as áreas de mata natural e de monoculturas.

No que se refere ao qMIC, pode-se verificar que na área de SAF-2 os valores foram mais elevados no

Tabela 3. Carbono da biomassa microbiana (C-BMS), respiração basal do solo e os quocientes microbiano ( $q$ MIC) e metabólico $\left(q \mathrm{CO}_{2}\right)$ dos solos sob dois sistemas agroflorestais (SAFs 1 e 2), mata secundária e agricultura anual (AgAn), na camada de 0-10 cm, em duas estações (inverno-I; verão-V).

Table 3. Microbial biomass carbon (C-MBC), soil basal respiration and the ratios microbial (qMIC) and metabolic $\left(\mathrm{qCO}_{2}\right)$ of soils under two agroforestry (SAF 1 and 2), secondary forest and annual agricultural (AnAg) in the layer of 0-10 cm, in two seasons (winter-I; summer-V). E = season; AnAg = Annual Agriculture .

\begin{tabular}{|c|c|c|c|c|c|}
\hline Áreas & Estações & $\begin{array}{c}\text { C - BMS } \\
\left(\mu g g^{-1}\right)\end{array}$ & $\begin{array}{l}\text { Respiração basal } \\
\left(\mu \mathrm{g} \mathrm{C}-\mathrm{CO}_{2} \mathrm{~g}^{-1} \mathbf{h}^{-1}\right)\end{array}$ & $\begin{array}{c}q \mathrm{mic} \\
(\%)\end{array}$ & $\begin{array}{c}q \mathrm{CO}_{2} \\
\left(\mathrm{mg} \mathrm{C}-\mathrm{BMS} \mathrm{h}^{-1}\right.\end{array}$ \\
\hline \multirow[t]{2}{*}{ SAF 1} & Inverno & $282,16^{\mathrm{aA}}$ & $0,96^{\mathrm{aA}}$ & $1,06^{\mathrm{abA}}$ & $3,4^{\mathrm{aA}}$ \\
\hline & Verão & $254,35^{\mathrm{bA}}$ & $1,32^{\mathrm{bA}}$ & $0,94^{\mathrm{abA}}$ & $5,2^{\mathrm{aA}}$ \\
\hline \multirow[t]{2}{*}{ SAF 2} & Inverno & $306,98^{\mathrm{aA}}$ & $1,01^{\mathrm{aA}}$ & $1,50^{\mathrm{aA}}$ & $3,3^{\mathrm{aA}}$ \\
\hline & Verão & $241,3^{\mathrm{bA}}$ & $1,23^{\mathrm{bA}}$ & $1,01^{\mathrm{abB}}$ & $5,1^{\mathrm{aA}}$ \\
\hline \multirow[t]{2}{*}{ AgAn } & Inverno & $232,64^{\mathrm{aA}}$ & $0,47^{\mathrm{aB}}$ & $0,91^{\mathrm{bA}}$ & $2,0^{\mathrm{aA}}$ \\
\hline & Verão & $159,64^{\mathrm{bA}}$ & $0,86^{\mathrm{bA}}$ & $0,52^{\mathrm{bB}}$ & $5,4^{\mathrm{aA}}$ \\
\hline \multirow[t]{2}{*}{ Mata } & Inverno & $357,39^{\mathrm{aA}}$ & $1,15^{\mathrm{aB}}$ & $1,23^{\mathrm{abA}}$ & $3,2^{\mathrm{aB}}$ \\
\hline & Verão & $472,09^{\mathrm{aA}}$ & $3,95^{\mathrm{aA}}$ & $1,1^{\mathrm{aA}}$ & $8,4^{\mathrm{aA}}$ \\
\hline
\end{tabular}

$\mathrm{E}=$ estações; $\mathrm{AgAn}=$ agricultura anual. Letras minúsculas iguais numa mesma coluna representam igualdade estatística entre os tratamentos na mesma estação, enquanto letras maiúsculas iguais numa mesma coluna representam igualdade estatística entre as estações no mesmo tratamento segundo o teste $t$ com correção de Bonferroni $(p<0,05)$. 
inverno em relação ao verão. Além disso, em ambas as estações, esta área apresentou valores qMIC significativamente maiores que a área de AgAn (Tabela 3). Este fato pode estar relacionado com a qualidade da matéria orgânica, bem como a fatores microclimáticos proporcionados pela cobertura vegetal viva e morta sobre o solo, no SAF-2, os quais tendem a reduzir a temperatura do solo (variações mais estáveis e de menor amplitude) e a aumentar a retenção de umidade, o metabolismo respiratório, a população e a biomassa microbiana (Eira, 2011).

Os valores de $q \mathrm{CO}_{2}$ não apresentaram diferença estatística para a maioria das comparações; no entanto, na área de mata, houve um aumento significativo deste quociente do inverno para o verão (Quadro 3). Este padrão pode estar indicando um aumento no estresse, provavelmente pelo aumento de temperatura, a qual possivelmente promoveu um aumento da atividade da BMS pela maior atividade fisiológica dos vegetais e, consequentemente, uma maior quantidade de exsudatos radiculares (Chen et al., 2006), que são fonte de C prontamente mineralizável para a BMS (Souza et al., 2010).

Comparando-se os dados deste estudo com os obtidos por Alfaro Villatoro (2004) em sistemas agroflorestais com café na Guatemala, os teores de C-BMS e o qMIC encontrados neste estudo foram, de forma geral, inferiores e a RBS foi maior, resultando em um $q \mathrm{CO}_{2}$ muito mais elevado.

\subsection{Componentes da respiração do solo em campo}

Os resultados da medição da respiração do solo em campo não apresentaram diferença estatística entre áreas, em ambas as estações. Já em relação à sazonalidade, o fluxo de $\mathrm{CO}_{2}$ aumentou do inverno para o verão no SAF 2 (Tabela 4). Dias (2006), em áreas de floresta nativa da Amazônia, observou influência da sazonalidade na dinâmica do fluxo de $\mathrm{CO}_{2}$ do solo, sendo que os maiores fluxos foram obtidos no período chuvoso, indicando que a disponibilidade de água e a temperatura do solo foram os principais condicionadores da produção do $\mathrm{CO}_{2}$.

A área de mata foi a que apresentou mais raízes em todas as categorias, no inverno. No verão, houve um aumento nas raízes finas do SAF-2, tal como ocorrido para a quantidade de $\mathrm{CO}_{2}$ detectada. Verificou-se, ainda, que o SAF-2 apresentou densidade de raízes finas semelhante à área de mata e superior à área de AgAn, nesta estação. Em relação à densidade de raízes grossas no $\mathrm{SAF}-2$, houve uma diminuição do inverno para o verão, não havendo diferença significativa entre as áreas, no verão. As densidades de raízes totais foram superiores na área de mata quando comparada às das demais áreas, em ambas as estações, exceto em relação à AgAn, no verão (Tabela 4).

Tabela 4. Respiração do solo em campo (RS) e as variáveis associadas: raízes finas, grossas e totais do solo na profundidade de $0-10 \mathrm{~cm}$ sob a câmara, serapilheira no interior das câmaras e umidade nos solos sob dois sistemas agroflorestais (SAFs 1 e 2), mata secundária e agricultura anual (AgAn), em duas estações (inverno-I; verão-V).

Table 4. Field soil respiration (SR) and the associated variables: fine roots, thick and total soil depth of $0-10 \mathrm{~cm}$ under the camera, litter inside the cameras and soil moisture under two agroforestry (AFS 1 and 2), secondary forest and annual agricultural (AnAg) in two seasons (winter-I; summer-V). Sera = litter; AnAg = annual agricultural; $\mathrm{S}=$ season; US = soil moisture.

\begin{tabular}{|c|c|c|c|c|c|c|c|}
\hline \multirow{2}{*}{ Áreas } & \multirow{2}{*}{$\mathbf{E}$} & RS & Raízes finas & Raízes grossas & Raízes total & \multirow{2}{*}{$\begin{array}{c}\text { Sera } \\
\left(\mathbf{g ~ m}^{-2}\right)\end{array}$} & \multirow{2}{*}{$\begin{array}{l}\text { US } \\
(\%)\end{array}$} \\
\hline & & $\left(\mathrm{mg} \mathrm{C}-\mathrm{CO}_{2} \mathrm{~m}^{-2} \mathrm{~h}^{-1}\right)$ & \multicolumn{3}{|c|}{$\mathrm{g} \mathrm{m}^{-2}$ solo $0-10 \mathrm{~cm}$} & & \\
\hline \multirow[t]{2}{*}{ SAF 1} & I & $125,1^{\mathrm{aA}}$ & $7,93^{\mathrm{bA}}$ & $11,78^{\mathrm{bA}}$ & $19,7^{\mathrm{bA}}$ & $650,79^{\mathrm{aA}}$ & $27,61^{\mathrm{aA}}$ \\
\hline & $\mathrm{V}$ & $138,91^{\mathrm{aA}}$ & $15,94^{\mathrm{bcA}}$ & $20,61^{\text {aA }}$ & $36,55^{\mathrm{bA}}$ & $293,5^{\text {bB }}$ & $29,92^{\mathrm{abA}}$ \\
\hline \multirow[t]{2}{*}{ SAF 2} & I & $49,56^{\mathrm{aB}}$ & $15,8^{\mathrm{bB}}$ & $33,15^{\mathrm{bA}}$ & $48,95^{\mathrm{bA}}$ & $756,88^{\mathrm{aA}}$ & $25,49^{\mathrm{aA}}$ \\
\hline & $\mathrm{V}$ & $127,46^{\text {aA }}$ & $26,92^{\mathrm{abA}}$ & $11,46^{\mathrm{aB}}$ & $38,39^{\mathrm{bA}}$ & $556,6^{\mathrm{abA}}$ & $25,19^{\mathrm{bA}}$ \\
\hline \multirow[t]{2}{*}{ AgAn } & I & N.D. & $2,86^{\mathrm{bA}}$ & $12,26^{\mathrm{bA}}$ & $15,12^{\mathrm{bA}}$ & $492,59^{\mathrm{aA}}$ & $30,93^{\mathrm{aA}}$ \\
\hline & $\mathrm{V}$ & $106,99^{\mathrm{a}}$ & $3,65^{\mathrm{cA}}$ & $59,67^{\mathrm{aA}}$ & $63,33^{\mathrm{abA}}$ & $218,63^{\mathrm{bA}}$ & $32,85^{\mathrm{aA}}$ \\
\hline \multirow[t]{2}{*}{ Mata } & I & $89,64^{\mathrm{aA}}$ & $47,90^{\mathrm{aA}}$ & $110,86^{\mathrm{aA}}$ & $158,75^{\mathrm{aA}}$ & $476,66^{\mathrm{aB}}$ & $28,35^{\mathrm{aB}}$ \\
\hline & $\mathrm{V}$ & $165,79^{\mathrm{aA}}$ & $48,95^{\text {aA }}$ & $133,05^{\mathrm{aA}}$ & $182^{\mathrm{aA}}$ & $762,24^{\mathrm{aA}}$ & $32,03^{\mathrm{aA}}$ \\
\hline
\end{tabular}

Sera = serapilheira; AgAn = agricultura anual; $\mathrm{E}$ = estações; US = umidade do solo. Letras minúsculas iguais numa mesma coluna representam igualdade estatística entre os tratamentos na mesma estação, enquanto letras maiúsculas iguais numa mesma coluna representam igualdade estatística entre as estações no mesmo tratamento segundo o teste $t$ com correção de Bonferroni $(\mathrm{p}<0,05)$. 
O método de análise da regressão para avaliação da contribuição das raízes para a respiração total do solo proposto por Kucera \& Kirkham (1971) se enquadra entre os métodos de integração de componentes (Hanson et al., 2000), com a vantagem de medir o fluxo in situ e sem a perturbação do solo. A estimativa da contribuição das raízes para a respiração total pelo método de regressão só foi possível com os dados do verão, em razão da falta de dados de emissão no inverno.

Considerando-se todas as áreas, verificou-se que as raízes finas explicam a maior parte da variação na respiração total do solo, apresentando o maior $\mathrm{R}^{2}(0,2906)$ e também o maior coeficiente angular $(\mathrm{a}=0,0012)$, que representa a intensidade com que as variáveis estão correlacionadas. A influência positiva das raízes grossas, com elevados valores no coeficiente angular e no $\mathrm{R}^{2}$, se destacou no SAF-1, e a influência negativa da umidade, com elevado $\mathrm{R}^{2}$ e coeficiente angular negativo. O SAF-2 apresentou forte influência das raízes finas, concordando com o aumento de densidade e de fluxo observado nesta área e nesta estação. A serapilheira e a umidade apresentaram altos valores de $\mathrm{R}^{2}$ no SAF-2, sendo que o primeiro obteve coeficiente angular pouco expressivo. A AgAn apresentou elevado $\mathrm{R}^{2}$ para raízes grossas, apesar do baixo coeficiente angular. Tal fato ocorreu por influência de uma amostra que apresentou um tubérculo, conferindo elevada biomassa de raiz grossa, com respiração ligeiramente superior às outras amostras. A influência negativa da umidade na respiração do solo da AgAn também foi relevante. Na mata, as duas categorias de raízes se mostraram importantes, sendo as finas menos representativas e de contribuição mais intensa, e as raízes grossas indicaram melhor a intensidade da emissão, mas sua contribuição foi menos intensa. Em relação à umidade, a mata se comportou de forma contrária às outras áreas, apresentando elevada correlação positiva (Tabela 5).

A respiração do solo livre de raízes e estimada pelo método da regressão de Kucera \& Kirkham (1971) está representada na Tabela 5 pela variável independente (b). A partir da subtração da respiração total do solo e a respiração do solo livre de raízes obtidas pelo método da regressão, foi obtida a contribuição das raízes para a respiração total do solo (Figura 1). Desta forma, se observa que a mata possui a maior contribuição de raízes, totalizando $39,2 \%$ da respiração total do solo. O SAF-1 apresentou uma contribuição de 12,75\% das raízes, seguido da AgAn com 5,79\%, e do SAF-2 com 3,03\% (Figura 1).

Nakane et al. (1983) postularam que, em ecossistemas florestais com estoques de carbono do solo em equilíbrio dinâmico, a contribuição das raízes para a respiração total do solo tende a $50 \%$,

Tabela 5. Regressão múltipla das variáveis explicativas raízes finas, raízes grossas, raízes totais, serapilheira e umidade com a variável de resposta respiração do solo em campo sob dois sistemas agroflorestais (SAFs 1 e 2), agricultura anual (AgAn) e mata secundária. Equação da reta $(y=a x+b)$, em que $(a)=$ coeficiente angular.

Table 5. Multiple regression of the explanatory variables fine roots, thick roots, total roots, litter and moisture with the response variable field soil respiration under two agroforestry (AFS 1 and 2), annual agriculture (AnAg) and secondary forest. Straight line equation $(y=a x+b)$, where: $(a)=$ angular coefficient.

\begin{tabular}{llllll} 
Áreas & \multicolumn{1}{c}{ Raízes finas } & \multicolumn{1}{c}{ Raízes grossas } & \multicolumn{1}{c}{ Raízes totais } & \multicolumn{1}{c}{ Serapilheria } & Umidade \\
SAF 1 & $\mathrm{y}=-0,0004 \mathrm{x}+0,012$ & $\mathrm{y}=0,0006 \mathrm{x}+0,0107$ & $\mathrm{y}=0,0006 \mathrm{x}+0,0101$ & $\mathrm{y}=-1 \mathrm{E}-05 \mathrm{x}+0,0146$ & $\mathrm{y}=-0,0006 \mathrm{x}+0,0286$ \\
& $\mathrm{R}^{2}=0,0181$ & $\mathrm{R}^{2}=0,3831$ & $\mathrm{R}^{2}=0,3503$ & $\mathrm{R}^{2}=0,3048$ & $\mathrm{R}^{2}=0,5215$ \\
SAF 2 & $\mathrm{y}=0,0013 \mathrm{x}+0,0082$ & $\mathrm{y}=-0,0008 \mathrm{x}+0,0112$ & $\mathrm{y}=0,0001 \mathrm{x}+0,0103$ & $\mathrm{y}=4 \mathrm{E}-06 \mathrm{x}+0,0081$ & $\mathrm{y}=-0,0004 \mathrm{x}+0,021$ \\
& $\mathrm{R}^{2}=0,0596$ & $\mathrm{R}^{2}=0,0275$ & $\mathrm{R}^{2}=0,0015$ & $\mathrm{R}^{2}=0,5242$ & $\mathrm{R}^{2}=0,3535$ \\
AgAn & $\mathrm{y}=0,0005 \mathrm{x}+0,0088$ & $\mathrm{y}=0,0001 \mathrm{x}+0,0085$ & $\mathrm{y}=0,0001 \mathrm{x}+0,0084$ & $\mathrm{y}=9 \mathrm{E}-07 \mathrm{x}+0,0087$ & $\mathrm{y}=-0,0002 \mathrm{x}+0,0161$ \\
& $\mathrm{R}^{2}=0,0019$ & $\mathrm{R}^{2}=0,6154$ & $\mathrm{R}^{2}=0,6106$ & $\mathrm{R}^{2}=0,0103$ & $\mathrm{R}^{2}=0,1841$ \\
Mata & $\mathrm{y}=0,001 \mathrm{x}+0,0105$ & $\mathrm{y}=0,0006 \mathrm{x}+0,0083$ & $\mathrm{y}=0,0004 \mathrm{x}+0,0084$ & $\mathrm{y}=7 \mathrm{E}-06 \mathrm{x}+0,0086$ & $\mathrm{y}=0,0011 \mathrm{x}-0,022$ \\
& $\mathrm{R}^{2}=0,1014$ & $\mathrm{R}^{2}=0,2085$ & $\mathrm{R}^{2}=0,1933$ & $\mathrm{R}^{2}=0,0779$ & $\mathrm{R}=0,1467$ \\
Todos & $\mathrm{y}=0,0012 \mathrm{x}+0,0092$ & $\mathrm{y}=0,0002 \mathrm{x}+0,0103$ & $\mathrm{y}=0,0002 \mathrm{x}+0,0099$ & $\mathrm{y}=5 \mathrm{E}-06 \mathrm{x}+0,0089$ & $\mathrm{y}=-9 \mathrm{E}-05 \mathrm{x}+0,014$ \\
& $\mathrm{R}^{2}=0,2906$ & $\mathrm{R}^{2}=0,1797$ & $\mathrm{R}^{2}=0,2466$ & $\mathrm{R}^{2}=0,2641$ & $\mathrm{R}^{2}=0,0128$ \\
\hline
\end{tabular}




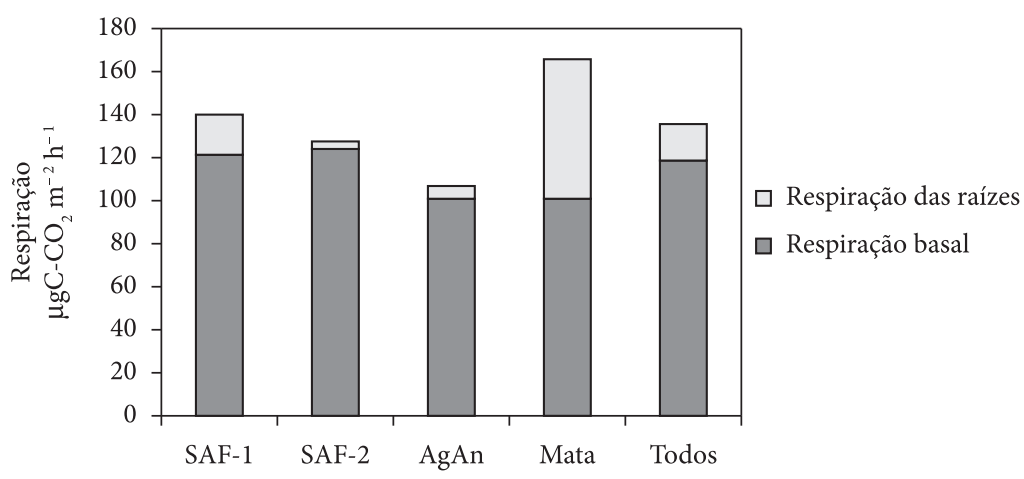

Figura 1. Contribuição das raízes para a respiração total do solo sob dois sistemas agroflorestais (SAFs 1 e 2), agricultura anual (AgAn) e mata secundária, estimada pelo método de regressão de Kucera \& Kirkham (1971).

Figure 1. Contribution of roots to total soil respiration under two agroforestry (AFS 1 and 2), annual agriculture (AnAg) and secondary forest, estimated by regression Kucera \& Kirkham (1971).

mesmo em condições climáticas distintas. Neste estudo, as contribuições da mata secundária foram próximas à destacada por esses autores. De acordo com Stevenson (1982), a respiração heterotrófica está associada ao processo de decomposição e depende dos estoques de carbono do solo. Em áreas agrícolas, embora os estoques de $\mathrm{C}$ sejam mais baixos e a densidade de raízes seja reduzida, a respiração do solo é estimulada pelo revolvimento do solo. Esta é a situação encontrada no presente estudo, em que as áreas de cultivo apresentam menor densidade e menor contribuição das raízes para a respiração total, predominando a respiração heterotrófica dos estoques de carbono do solo, enquanto, na mata secundária, a colonização de raízes é mais intensa e a contribuição das raízes é maior e mais próxima de $50 \%$ do que nas outras áreas.

\section{CONCLUSÕES}

O manejo de poda nos sistemas agroflorestais é capaz de promover aumento nos teores e/ou nos estoques de nitrogênio, fósforo, potássio e magnésio da serapilheira, levando à condição de semelhança aos estoques da mata e de superioridade em relação aos estoques da AgAn.

O carbono da biomassa microbiana do solo e a respiração basal não variaram entre os SAFs e a área de AgAn. Em contrapartida, no verão, a mata apresentou os maiores valores destas variáveis, aliados ao conteúdo de carbono orgânico total, indicando maior equilíbrio natural em termos de desenvolvimento das plantas.

O SAF-2 apresentou elevado quociente microbiano no inverno, demonstrando maior sensibilidade dos microrganismos às mudanças de cobertura vegetal do que aos atributos químicos do solo.

\section{STATUS DA SUBMISSÃO}

Recebido: 24/04/2012

Aceito: 22/09/2012

Publicado: 31/12/2012

\section{AUTOR(ES) PARA CORRESPONDÊNCIA}

\section{Miguel Seabra Correa da Silva}

Instituto de Desenvolvimento e Ação

Comunitária - IDACO, CEP 20091-007,

Rio de Janeiro, RJ, Brasil

e-mail: migscs@hotmail.com

\section{Eliane Maria Ribeiro da Silva}

Embrapa Agrobiologia, Rod. BR 465, Km 7, CEP 23890-000, Seropédica, RJ, Brasil e-mail: eliane@cnpab.embrapa.br

\section{Marcos Gervasio Pereira}

Departamento de Solos, Universidade Federal Rural do Rio de Janeiro - UFRRJ, Rod. BR 465, Km 7, CEP 23890-000, Seropédica, RJ, Brasil e-mail: mgervasiopereira01@gmail.com 


\section{Cristiane Figueira da Silva}

Departamento de Solos, Universidade Federal Rural do Rio de Janeiro - UFRRJ, Rod. BR 465, Km 7, CEP 23890-000, Seropédica, RJ, Brasil e-mail: cfigueirasilva@yahoo.com.br

\section{APOIO FINANCEIRO}

\section{CAPES}

\section{REFERENNCIAS}

Alfaro Vilatoro MA. Matéria orgânica e indicadores microbiológicos da qualidade do solo na cultura de café sob manejo agroflorestal e orgânico [tese]. Seropédica: Universidade Federal Rural do Rio de Janeiro; 2004.

Albrecht A, Kandji ST. Carbon sequestration in tropical agroforestry systems. Agriculture, Ecosystems and Environment 2003; 99: 15-27. http://dx.doi.org/10.1016/ S0167-8809(03)00138-5

Anderson TH, Domsch $\mathrm{KH}$. Application of ecophysiological quotients ( $\mathrm{qCO}_{2}$ and $\mathrm{qD}$ ) on microbial biomasses from soils of different cropping histories. Soil Biology and Biochemistry 1990; 22: 251-255. http:// dx.doi.org/10.1016/0038-0717(90)90094-G

Anderson TH, Domsch KH. Determination of ecophysiological maintenance requirements of soil microorganisms in a dormant stage. Biology and Fertility of Soils 1985; 11: 81-89.

Assis-Júnior SL, Zanuncio JC, Kasuya MCM, Couto L, Melido RCN. Atividade microbiana do solo em sistemas agroflorestais, monoculturas, mata natural e área desmatada. Revista Árvore 2003; 27: 35-41.

Bailey BJR. Tables of the Bonferroni t statistic. Journal of the American Statistical Association 1977; 72: 469-472.

Bartlett RJ, Ross DS. Colorimetric determination of oxidizable carbon in acid soil solutions. Soil Science Society of America Journal 1988; 52: 1191-1192. http:// dx.doi.org/10.2136/sssaj1988.03615995005200040055x

Brasil. Ministério das Minas e Energia. Secretária Geral. Projeto RADAMBRASIL. Levantamentos dos Recursos Naturais. Rio de Janeiro, 1983. 775 p. v. 32. Folhas S. $23 / 24$.

Chen Y, Lee P, Lee G, Mariko S, Oikawa T. Simulating root responses to grazing of a Mongolian grassland ecosystem. Plant Ecology 2006; 183: 265-275. http:// dx.doi.org/10.1007/s11258-005-9038-7

Cidin ACM, Corrêa FLO, Pequeno PLL, Almeida CMVC, Müller MW, Macedo RGM et al. Avaliação da fertilidade do solo em sistema agroflorestal com cacaueiros e coqueiros em Ji-Paraná, Rondônia, Brasil. Agrotrópica 2009; 21(1): 65-72.
Dias JD. Fluxo do $\mathrm{CO}_{2}$ proveniente da respiração do solo em áreas de floresta nativa da Amazônia [dissertação]. Piracicaba: Escola Superior de Agricultura Luiz de Queiroz; 2006.

Eira AF. Influência da cobertura morta na biologia do solo. [cited 2011 fev. 15]. Available from: www.ipef.br/ publicacoes/seminario_cultivo_minimo/cap03.pdf.

Ferreira DF. Sisvar. versão 4.2. DEX/UFLA; 2003.

Embrapa. Manual de métodos de análise de solos. Rio de Janeiro: CNPS; 1997.

Jenkinson DS, Rayner JH. The turnover of organic matter in some of the Rothamsted Classical Experiments. Soil Science 1977; 123: 298-305. http:// dx.doi.org/10.1097/00010694-197705000-00005

Jenkinson DS, Ladd JN. Microbial biomass in soil: measurement and turnover. In: Paul EA, Ladd JN, editors. Soil Biochemistry. New York: Marcel Dekker; 1981. vol. 5, p. 415-471.

Jenkinson DS, Powlson DS. The effects of biocidal treatments on metabolism in soil-I. Fumigation with chloroform. Soil Biology and Biochemestry 1976; 8: 167-177. http://dx.doi. org/10.1016/0038-0717(76)90001-8

Hanson PJ, Edwards NT, Garden CT, Andrews JA. Separating root and soil microbial contributions to soil respiration: A review of methods and observations. Biogeochemistry 2000; 48: 115-146.

Kucera CL, Kirkham DL. Soil respiration studies in tall grass prairies in Missouri. Ecology 1971; 52: 912-915. http://dx.doi.org/10.2307/1936043

Lundegardh A. Carbon dioxide production from soil and crop growth. Soil Science 1927; 23: 417-453.

Macdicken KG, Vergara NT. Introduction to agroforestry. In: Macdicken KG, Vergara NT, editors. Agroforestry: classification and management. New York: John Wiley Sons; 1990.

Mendonça ES, Leite LFC, Ferreira Neto PS. Cultivo de café em sistema agroflorestal: uma opção para a recuperação de solos degradados. Revista Árvore 2001; 25: 375-383.

Menezes JMT, Van Leeuwen J, Valeri SV, Cruz MCP, Leandro RC. Comparação entre solos sob uso agroflorestal e em florestas remanescentes adjacentes, no norte de Rondônia. Revista Brasileira de Ciência do Solo 2008; 32: 893-898. http://dx.doi.org/10.1590/ S0100-06832008000200043

Nakane K, Yamamoto M, Tsubota H. Estimation of root respiration rate in a mature forest ecosystem. Japanese Journal of Ecology 1983; 33: 397-408.

Powlson DS, Brookes PC, Christensen BT. Measurement of soil microbial biomass provide an early indication of changes in total soil organic 
matter due to straw incorporation. Soil Biology and Biochemistry 1987; 19: 159-164. http://dx.doi. org/10.1016/0038-0717(87)90076-9

Reinert DJ. Recuperação de solos em sistemas agropastoris. In: Dias LE, Mello JWV, editores. Recuperação de áreas degradadas. Viçosa: UFV, SOBRADE; 1998.

Schlesinger WH, Andrews JA. Soil respiration and the carbon cycle. Biogeochemistry 2000; 48:7-20. http:// dx.doi.org/10.1023/A:1006247623877

Schlesinger WH. Biogeochemistry: Analysis of global change. 2rd ed. Oxon: Academic Press; 1997.

Silva EE, Azevedo PHS, De-Polli H. Determinação de Respiração Basal (RBS) e quociente metabólico do solo $\left(q \mathrm{CO}_{2}\right)$. Embrapa; 2007. p. 4. Comunicado Técnico, no. 99 .

Souza ED, Costa SEVGA, Anghinoni I, Lima CVS, Carvalho PCF, Martins AP. Biomassa microbiana do solo em sistema de integração lavoura-pecuária em plantio direto, submetido a intensidades de pastejo. Revista Brasileira de Ciência do Solo 2010; 34: 79-88. http://dx.doi.org/10.1590/S0100-06832010000100008

Stevenson FJ. Humus chemistry: genesis, composition, reactions. New York: John Wiley; 1982.

Vance ED, Brookes PC, Jenkinson DS. An extraction method for measuring soil microbial biomass C. Soil Biology and Biochemistry 1987; 19: 703-707. http:// dx.doi.org/10.1016/0038-0717(87)90052-6 